\title{
Regulation of vascular smooth muscle growth by cyclic nucleotides and cGMP-dependent protein kinase Pascal Weinmeister*1, Robert Lukowski ${ }^{1}$, Stefan Linder ${ }^{2}$, Wolfgang Erl2 ${ }^{2}$ Richard Brandl $^{3}$, Susanne Feil ${ }^{1}$, Franz Hofmann ${ }^{1}$ and Robert Feil ${ }^{1}$
}

\author{
Address: ${ }^{1}$ Institut für Pharmakologie und Toxikologie, TU München, Germany, ${ }^{2}$ Institut für Prophylaxe und Epidemiologie der \\ Kreislaufkrankheiten, LMU München, Germany and ${ }^{3}$ Krankenhaus München Schwabing, Gefäßchirurgie, Germany \\ Email: Pascal Weinmeister* - Weinmeister@ipt.med.tu-muenchen.de \\ * Corresponding author
}

from 2nd International Conference of cGMP Generators, Effectors and Therapeutic Implications

Potsdam, Germany, 10-12 June, 2005

Published: 16 June 2005

BMC Pharmacology 2005, 5(Suppl I):P62 doi:10.1186/I47I-2210-5-SI-P62

The role of cGMP and cGMP-dependent protein kinase type I (cGKI) in the phenotypic modulation and growth of vascular smooth muscle cells (VSMCs) is not well understood. The general view, which is mainly based on the in vitro analysis of subcultured (repeatedly passaged) cells, is that activation of the cGMP/cGKI pathway inhibits the proliferation of VSMCs. In contrast, recent in vivo studies with transgenic mice indicated that cGMP/cGKI signaling in VSMCs promotes vasculo-proliferative processes. Here, we have studied the effects of various "cGK agonists" (8-Br-cGMP， 8-pCPT-cGMP， 8-Br-PET-cGMP) and "cGK inhibitors" (Rp-8-pCPT-cGMPs, Rp-8-Br-PETcGMPs, DT-2) on the growth of primary aortic VSMCs from control and cGKI-deficient mice. All tested agonists stimulated the growth of primary VSMCs via activation of cGKI, whereas the tested "cGK inhibitors" failed to inhibit cGKI activity and cell growth. 8-Br-cAMP, an activator of the cAMP-dependent protein kinase ( $\mathrm{CAK})$, inhibited the growth of both wild-type and cGKI-deficient cells. These results suggest that (i) activation of the CGMP/CGKI pathway stimulates the growth of primary VSMCs, (ii) "cGKI inhibitors" should be used with caution in intact VSMCs, and (iii) CAMP inhibits VSMC growth by an cGKI-independent mechanism. Interestingly, the growth of various subcultured VSMC lines (obtained from rat and human media and intima) was barely affected by cGMP, but strongly inhibited by cAMP. Taken together, these results indicate that the effects of cGMP on VSMC growth differ between primary and subcultured cells. In primary VSMCs, cGMP stimulates growth via activation of cGKI. In contrast, subcultured cells appear to be refractory to growth stimulation by cGMP and cGKI, perhaps because repeated passaging results in dysregulation of the CGMP/ cGKI pathway or in more general alterations of proliferative responses. The reported anti-proliferative effect of cGMP analogs on subcultured VSMCs might be caused by cross-activation of the CAMP/CAK pathway. 